\title{
Reduced attention-driven auditory sensitivity in hallucination-prone individuals
}

\author{
Louise H. Rayner, Kwang-Hyuk Lee and Peter W. R. Woodruff
}

\section{Background}

Evidence suggests that auditory hallucinations may result from abnormally enhanced auditory sensitivity.

\section{Aims}

To investigate whether there is an auditory processing bias in healthy individuals who are prone to experiencing auditory hallucinations.

\section{Method}

Two hundred healthy volunteers performed a temporal order judgement task in which they determined whether an auditory or a visual stimulus came first under conditions of directed attention ('attend-auditory' and 'attend-visual' conditions). The Launay-Slade Hallucination Scale was used to divide the sample into high and low hallucinationproneness groups.

\section{Results}

The high hallucination-proneness group exhibited a reduced sensitivity to auditory stimuli under the attend-auditory condition. By contrast, attention-directed visual sensitivity did not differ significantly between groups.

\section{Conclusions}

Healthy individuals prone to hallucinatory experiences may possess a bias in attention towards internal auditory stimuli at the expense of external sounds. Interventions involving the redistribution of attentional resources would have therapeutic benefit in patients experiencing auditory hallucinations.

\section{Declaration of interest}

None.

\section{Copyright and usage}

(c) The Royal College of Psychiatrists 2015.
Auditory hallucinations are one of the most commonly experienced and distressing symptoms of schizophrenia, but can also occur in the healthy population. ${ }^{1}$ Recent evidence suggests that auditory hallucinations may result from abnormally enhanced sensitivity of the auditory regions of the brain to external auditory stimuli. In particular, this may arise when individuals are paying specific attention to auditory signals. ${ }^{2,3}$ We aimed to test whether hallucination-prone individuals would show a bias towards auditory signals during audiovisual perception when directing attention to stimuli in the auditory modality. We predicted that highly hallucination-prone individuals would display enhanced sensitivity to auditory stimuli under conditions of auditory attention in comparison with individuals who are less prone to having hallucinatory experiences.

\section{Method}

\section{Participants}

Two hundred healthy volunteers participated, the majority of whom were students or staff at the University of Sheffield. The sample comprised 89 males and 111 females, with a mean age of 26.49 years $($ s.d. $=12.22$; range $=18-68$ ). All participants declared normal or corrected-to-normal sight and none declared any hearing impairments. Visual acuity was not formally assessed. No participants reported any past or current history of psychiatric or neurological disorders. Written informed consent was obtained from all participants prior to their participation. This study was approved by the University of Sheffield Medical School Research Ethics Committee.

\section{Materials and apparatus}

All participants performed an audiovisual temporal order judgement (TOJ) task. Data were collected using a Toshiba Satellite Pro A300 laptop, using 'Presentation version 14.9' software to run the task. ${ }^{4}$ Responses were made using the left and right arrow keys on the laptop keyboard. The auditory stimuli were presented using Sennheiser HD 202 headphones. Throughout the task, participants were seated approximately $45 \mathrm{~cm}$ from the computer screen. All participants were assessed by the first author in a quiet room with minimal external noise and visual distractions in order to ensure the uniformity of testing conditions for all participants.

\section{Task stimuli and experimental procedure}

The audiovisual TOJ task was performed by all participants to provide a measure of their sensitivity to auditory and visual stimuli under attention-driven conditions. The auditory stimulus was a $1000 \mathrm{~Hz}$ tone delivered through headphones, at an approximate sound-pressure level of 65 decibels. The visual stimulus was a white circle of $60 \mathrm{~mm}$ in diameter, which was presented at the centre of the laptop computer screen on a black background in front of the participant. The duration of both stimuli was $20 \mathrm{~ms}$.

All patients participated in two experimental conditions, an 'attend-auditory' condition and an 'attend-visual' condition. In the task, participants' attention was focused on either the auditory or the visual sensory modality by altering the task instructions. For the 'attend-auditory' condition, participants were instructed to "Click "yes" when the bleep comes first and "no" when it doesn't'. For the 'attend-visual' condition, participants were instructed to "Click "yes" when the flash comes first and "no" when it doesn't'. In this way, we aimed to implicitly manipulate participants' attention, thus reducing the possibility that responses would be biased by explicit instructions to attend to a particular sensory modality.

The audiovisual stimulus pairs were presented at 11 different stimulus onset asynchronies (SOAs): $-240,-120,-90,-60$, $-30,0,30,60,90,120$ and $240 \mathrm{~ms}$. This range of SOAs was the same as in previous studies. ${ }^{5}$ The SOA represents the interval, in milliseconds, between the onsets of the two stimuli. Here, the negative values indicate that the attended stimulus was presented 
first (before the unattended stimulus), and the positive values indicate that the attended stimulus was presented second (after the unattended stimulus). Responses were made using the left and right arrows of the laptop keyboard. Participants had $3.5 \mathrm{~s}$ to respond before the next stimulus pair was presented and were instructed to respond as quickly and accurately as possible.

Prior to commencing the task, participants completed a practice block for each of the experimental conditions. The practice block consisted of five pairs of stimuli at SOAs of $-240,-120,120$ and 240 ms. Feedback was given to participants during the practice blocks as to whether they had responded correctly or incorrectly.

In the testing phase, the task comprised six blocks (three for each attention condition), which each consisted of 110 stimulus pairs. Task block order was either AVAVAV or VAVAVA for each participant, where ' $A$ ' represents the 'attend-auditory' condition and ' $\mathrm{V}$ ' represents the 'attend-visual' condition. Task block order was alternated for each successive participant.

In total, there were 30 trials for each attentional condition for each of the 11 SOAs (660 trials in total). Each task block lasted approximately $8 \mathrm{~min}$. Participants were encouraged to take breaks between the task blocks, and were informed that they could 'pause' the task at any time if required to maintain their concentration. The total testing time was approximately $45 \mathrm{~min}$.

\section{Questionnaires}

Participants completed the revised Launay-Slade Hallucination Scale (LSHS-R) ${ }^{6,7}$ which provides a measure of general hallucinatory predisposition in non-clinical samples. The scale was constructed using items related to clinical symptoms such as auditory and visual hallucinations, and possible subclinical forms such as intrusive thoughts and vivid daydreams. Higher scores indicate higher hallucination proneness. The LSHS-R allowed for the subsequent division of the sample into high and low hallucination-proneness groups.
To examine whether our measure was specific to auditory hallucinations or other schizotypal personality traits, we administered the Schizotypal Personality Questionnaire (SPQ), a measure of schizotypal personality traits based on the DSM-III-R diagnostic criteria for schizotypal personality disorder. The SPQ tests for traits related to the nine features of the DSM-III-R diagnostic criteria for schizotypal personality disorder: ideas of reference, excessive social anxiety, odd beliefs, unusual perceptual experiences, eccentric behaviour, no close friends, odd speech, constricted affect and suspiciousness.

Given the potential for the underreporting of psychiatric symptoms in a university population, a 21 -item Lie Scale, taken from the Eysenck Personality Questionnaire, ${ }^{9}$ was randomly integrated in the SPQ. Use of the Lie Scale scores enabled us to control for underreporting of psychological difficulties when analysing the data. Illicit drug use and current medication (if applicable) were also recorded. Finally, participants were screened for potential hearing impairments using the Hearing Handicap Inventory for Adults (HHIA). ${ }^{10}$

\section{Data analysis}

Proportions of 'yes' responses at each SOA for both attentional conditions were calculated for every individual and displayed as a psychophysical function (Fig. 1). These functions showed a sigmoidal pattern that increased gradually at first, more rapidly in the middle, and slowly towards the end. The following model (the Morgan-Mercer-Flodin family function) was shown to be the best fit for the individual data, using the curve fitting software, CurveExpert 1.4. ${ }^{11}$

$$
f(x)=\frac{a b+c x^{d}}{b+x^{d}}
$$

where $y$ was proportion of 'yes' responses and $x$ was SOA. The four parameters in this model $(a-d)$ were initially not weighted in accordance with previous studies. ${ }^{12}$

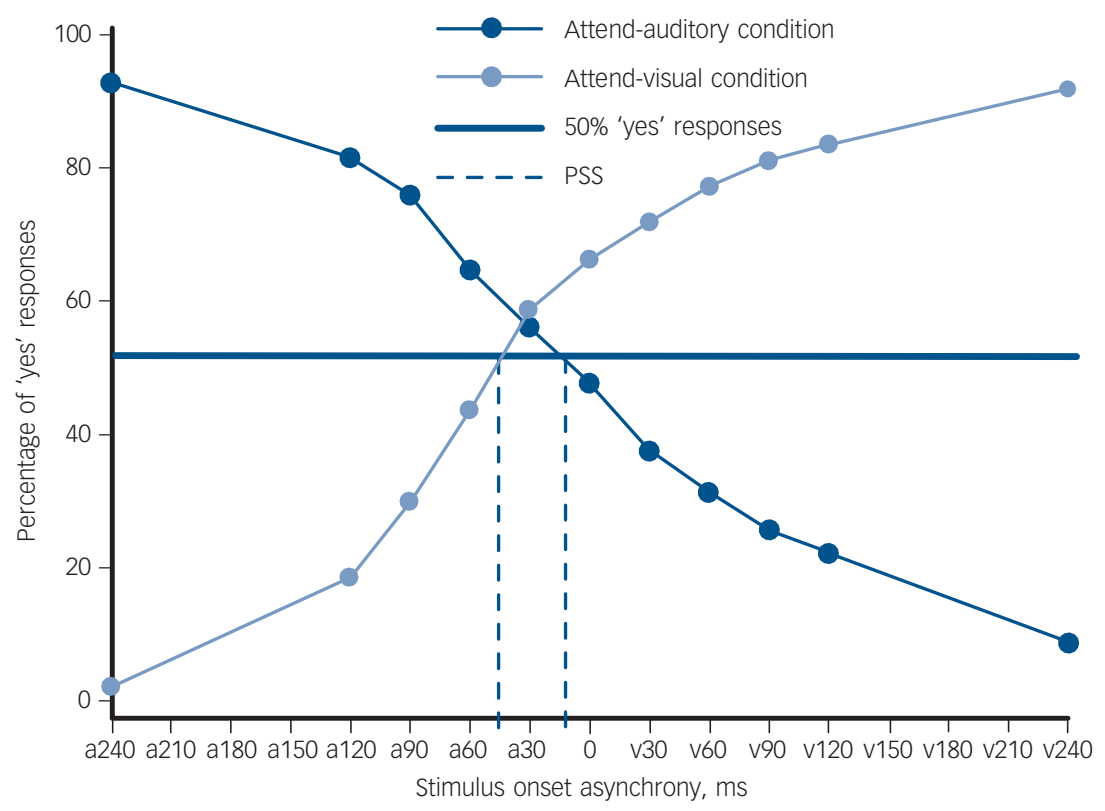

Fig. 1 The proportion of 'yes' responses at each stimulus onset asynchrony (SOA) for both the attend-auditory and attend-visual conditions. The dark blue circular markers represent the mean percentage of 'yes' responses at each SOA for the attend-auditory condition in which participants were asked to 'click "yes" when the tone comes first and "no" when it doesn't'. The light blue circular markers represent the mean percentage of 'yes' responses for each sOA under the attend-visual condition, in which participants were asked to 'click "yes" when the circle comes first and "no" when it doesn't'. The $x$-axis shows the SOA between the stimuli. On the $x$-axis, 'a' demonstrates that the auditory stimulus was presented before the visual stimulus and ' $v$ ' indicates that the visual stimulus was presented before the auditory. The $y$-axis represents the percentage of 'yes' responses for each condition. The intersection of the attend-auditory and attend-visual lines with the bold blue line ( $50 \%$ 'yes' responses) represents the point of subjective simultaneity (PSS) for each condition, further indicated by the blue dashed lines. 
We calculated the point of subjective simultaneity (PSS) for each attentional condition in each individual. The PSS represents the SOA at which a participant is equally likely to perceive either stimulus as first (50\% 'yes' responses) and hence can be calculated by taking the value of the function when $y=50$.

Given that positive SOAs indicate that the unattended stimulus was presented before the attended stimulus, the PSS value increases as the processing speed of the attended stimulus relative to the unattended stimulus increases. For example, in the attend-auditory condition, a PSS of $+50 \mathrm{~ms}$ means that the participant perceived the stimuli to be simultaneous when the visual stimulus was presented $50 \mathrm{~ms}$ before the auditory stimulus. Thus, when attending to a particular sensory modality, the higher the PSS, the greater the attention-driven sensitivity towards stimuli presented in that sensory modality.

In accordance with previous studies, ${ }^{13-15}$ participants were excluded from the analysis for one of four reasons: if the PSS value or the 25th or 75 th percentiles of their psychophysical function could not be calculated, if their 25th and 75th percentile values were less than $-480 \mathrm{~ms}$ or greater than $+480 \mathrm{~ms}$, if very poor concentration was observed by the examiner or reported by the participant, or if participants scored higher than 20 on the HHIA, indicating poor hearing acuity. We did not collect any information on short-term memory or other higher cognitive abilities, as working memory has been shown not to be associated with TOJ performance. ${ }^{16}$

\section{Results}

\section{Sample characteristics}

Of the 200 healthy volunteers who participated in the study, 46 participants were excluded (19 males, mean age 28.23 years). Forty-four participants were excluded as they were unable to perform the TOJ task with a sufficiently high level of accuracy, according to the criteria listed above. One further participant was excluded from the data analysis because of a hearing impairment and another was excluded because of an experimental error. The mean age of the remaining sample was 25.97 (s.d. $=11.95$, range 18-68; 70 males, 84 females).

Overall, excluded participants had higher mean SPQ scores than those who were included, a finding which approached significance $(U=2887.0, P=0.057)$. Exclusion from the analysis was not related to age $(t=-1.107, P=0.270)$, gender $\left(\chi^{2}(1\right.$ d.f. $)=0.247, P=0.619)$, use of illicit drugs $\left(\chi^{2}(1\right.$ d.f. $)=1.865$, $P=0.172)$ or medication $\left(\chi^{2}(1\right.$ d.f. $\left.)=0.108, P=0.742\right)$.

\section{The effect of attention on stimulus perception}

Participants' data were analysed as a whole to determine whether or not the prior entry effect had been elicited from patients. The mean attend-auditory PSS for the sample was $-1.97 \mathrm{~ms}$ (s.d. $=54.88)$ and the mean attend-visual PSS was $57.45 \mathrm{~ms}$ (s.d.=61.15), and they were statistically different (Wilcoxon signed-rank test: $Z=7.698, \quad P<0.001)$. This is displayed graphically in Fig. 1.

\section{The LSHS-R groups revealed in the cluster analysis}

A hierarchical agglomerative cluster analysis using Ward's method was performed on the LSHS-R data to separate participants based on their level of hallucination proneness. Analysis of the agglomeration schedule revealed an inconsistent increase in variance in the transition between the agglomeration coefficients for a two-cluster solution and a three-cluster solution. A two-cluster solution was therefore shown to be the optimal solution as succeeding clusters added much less to distinguishing between cases. Cluster number and membership were shown to be stable when the analysis was repeated on a random half of the sample.

The first cluster consisted of 63 participants with a mean LSHS-R score of 21.13 (s.d.=5.46). This represents the highscoring participants. The other cluster contained 91 participants, with a mean LSHS-R score of 7.64 (s.d.=3.75), representing the low scoring individuals. There was no significant difference between clusters: in age $(t=-0.478, P=0.633)$, gender $\left(\chi^{2}(1\right.$ d.f. $)=0.044, P=0.834)$, use of illicit drugs $\left(\chi^{2}(1\right.$ d.f. $)=0.713$, $P=0.398)$, medication $\left(\chi^{2}(1\right.$ d.f. $\left.)=1.185, P=0.276\right)$ or hearing acuity $(U=2700.5, P=0.371)$.

\section{Differences in attention-driven auditory sensitivity between the high and low hallucination-proneness groups}

As shown in Fig. 2, the highly hallucination-prone group had lower mean attend-auditory PSS values compared with the lower hallucination-proneness group $(U=2320.0, P=0.045)$, indicating reduced attention-driven auditory sensitivity in the high hallucination-proneness group. By contrast, attend-visual PSS did not differ significantly between the groups $(U=2848.0$, $P=0.946$ ). There were no significant differences in the slope of psychophysical functions between groups.

\section{Attention-driven auditory sensitivity and schizotypal personality traits}

A trend-level positive correlation was observed between attendauditory PSS and total SPQ score $(r=0.136, P<0.1)$. Furthermore, attend-auditory PSS values were positively correlated with the SPQ subscales: 'Eccentric Behaviour' (Spearman's $\rho=0.190, P=0.018$ ), 'No Close Friends' (Spearman's $\rho=0.200, P=0.013$ ) and 'Constricted Affect' (Spearman's $\rho=0.159, P=0.048$ ). After controlling for Lie Scale scores, the relationship between attend-auditory PSS and the SPQ subscales remained similar: 'Eccentric Behaviour' (Spearman's $\rho=0.146, P=0.073$ ), 'No Close Friends' (Spearman's $\rho=0.188, \quad P=0.020)$ and 'Constricted Affect' (Spearman's $\rho=0.137, P=0.092$ ). There was no relationship between attendvisual PSS and any of the SPQ subscales. These correlation results were not corrected for multiple comparisons.

\section{Discussion}

The main aim of this study was to investigate the relationship between attention-driven auditory sensitivity and hallucination proneness. We manipulated participants' attention implicitly via the task instructions during an audiovisual TOJ task. We found that the highly hallucination-prone group exhibited a reduced sensitivity to auditory stimuli under the attend-auditory condition, whereas there was no significant between-group difference in sensitivity to visual stimuli under the attend-visual condition. Our study provides direct experimental evidence for reduced sensitivity to external auditory signals in hallucinationprone individuals, and this finding may assist in understanding the mechanism of auditory hallucinations in schizophrenia, if replicated in a patient population.

\section{Hallucination proneness and attention-driven auditory sensitivity}

We found that highly hallucination-prone individuals have a reduced sensitivity to attention-driven auditory stimuli. This result contradicts our initial predication: individuals who were 


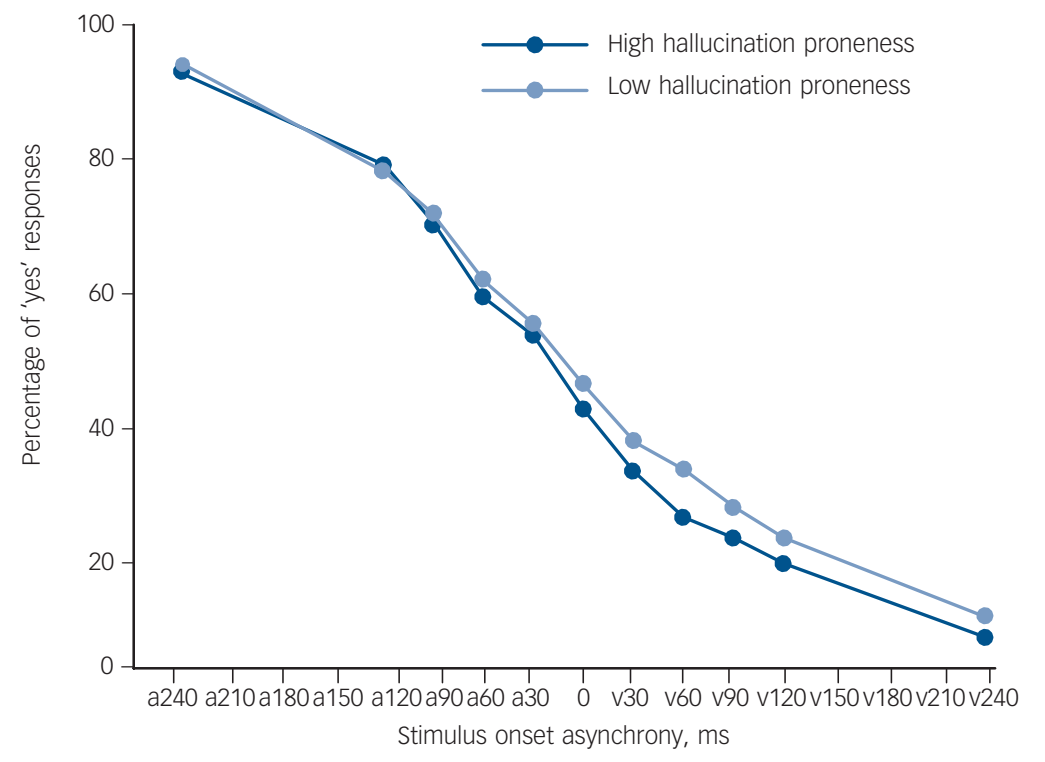

High hallucination proneness

Low hallucination proneness

Fig. 2 The proportion of 'yes' responses at each stimulus onset asynchrony (SOA) for both the attend-auditory in the high and low hallucination-proneness groups (left figure).

The dark blue circular markers represent the mean percentage of 'yes' responses at each SOA for the attend-auditory condition in the high hallucination-proneness group. The light blue circular markers represent the mean percentage of 'yes' responses for each SOA under the attend-auditory condition, in the low hallucination-proneness group. On the $x$-axis, ' $a$ ' demonstrates that the auditory stimulus was presented before the visual stimulus and ' $v$ ' indicates that the visual stimulus was presented before the auditory. The $y$-axis represents the percentage of 'yes' responses for each condition. The bar graph on the right shows the difference in mean attend-auditory point of subjective simultaneity (PSS) between the high and low hallucination-proneness groups. The high hallucination-proneness group had a mean attend-auditory PSS of 15.73 ms lower than that of the low hallucination-proneness group $(U=2320.0, P=0.045)$. The error bars represent the standard error of the mean.

prone to experiencing auditory hallucinations would display high attention-driven auditory sensitivity in comparison to those who were less prone to experiencing auditory hallucinations.

In light of this, we suggest the possibility that highly hallucination-prone individuals have an attentional bias towards internal brain-derived signals at the expense of the external environmental signals. Although this has not been empirically tested, it could account for our observation of reduced sensitivity to external auditory stimuli in hallucination-prone participants. Consequently, as we observed, hallucination-prone individuals may display a reduced sensitivity to external stimuli because of a relative inability to direct their attention away from internal signals. These internal signals may be spontaneous activations in the auditory cortex which have been shown to occur in the absence of external stimulation in healthy individuals. ${ }^{2}$ There was no difference in 'attend-visual' PSS between the two groups, indicating that our findings are specific to the auditory modality.

Our proposal concurs with previous studies involving patients with schizophrenia which have shown that internal and external auditory stimuli compete for processing at the level of the auditory cortex. Using functional magnetic resonance imaging, Woodruff et $a l^{17}$ observed that patients with schizophrenia demonstrated reduced activation in the auditory cortex to external speech compared with healthy controls. Furthermore, external speech was shown to activate the speech-processing regions of the brain significantly less when patients were severely hallucinating than when their hallucinations were remitted. In a similar study by Ford et al, ${ }^{18}$ decreased activation to pure tones was observed in the left primary auditory cortex in hallucinating patients compared with both non-hallucinating patients and healthy controls. In both studies, the authors concluded that auditory hallucinations may compete with external speech for processing in the temporal cortex. There is evidence that some hallucinations are triggered by degraded processing of external sounds. ${ }^{19}$ It is possible, therefore, that there might be complex interactions between internally and externally driven processes in their pathophysiology. Manipulation of acoustic features of voice stimuli in different attention conditions could be used to examine the interaction (between attention and auditory processing of specific speech elements) that might contribute to the experience of auditory hallucinations.

An alternative explanation for our results is that hallucinationprone individuals may have a tendency to focus on their thoughts instead of on the task stimuli and as such display poorer attention during the experiment overall. However, if this was the case, we would expect to see differences in the slope of the psychophysical functions between the high and low hallucination-proneness groups, representing differences in temporal sensitivity, which was not observed in this study. To our knowledge, this is the first behavioural study to examine attention-driven auditory sensitivity in healthy hallucination-prone individuals. Further research is therefore required to clarify and expand on our results.

\section{Schizotypal personality traits and attention-driven auditory sensitivity}

A trend-level relationship was observed between overall SPQ scores and attention-driven auditory sensitivity. When the SPQ subscales were assessed individually, the subscales 'Eccentric Behaviour', 'No Close Friends' and 'Constricted Affect' were associated with reduced attention-driven auditory sensitivity. No relationship was observed between auditory sensitivity and any of the other SPQ subscales, suggesting that these subscales were responsible for driving the trend observed in overall SPQ scores. This is an interesting finding, as these three traits may be associated with relatively more social isolation. Given that this sample comprised healthy volunteers, likely to have a reasonable social network, it would be important to test this hypothesis in patients who experienced social isolation as a result of their illness.

Hoffman et al $^{20}$ questioned 46 patients regarding their first experience of hearing 'voices' and $73 \%$ reported that the voices had emerged during a period of relative social isolation such 
as moving to a new area or travelling to a different country. Furthermore, Nayani \& David ${ }^{21}$ reported that $80 \%$ of patients with borderline personality disorder reported that being alone worsened their auditory hallucinations. It is, therefore, possible that individuals who often isolate themselves from others may be more inclined to attend to spontaneous brain activity. These internal activations, which are likely to be ignored in healthy non-hallucinating brains, may be over-interpreted in highly schizotypal individuals.

\section{Experimental issues}

Across the whole sample, a statistically significant difference was observed between the attend-auditory and attend-visual PSS values. Given that the stimulus pairs used in the attend-auditory and attend-visual conditions were identical, if attention had not affected stimulus perception, the PSS for both conditions would have occurred at the same SOA. A significant difference between the attend-auditory and attend-visual PSS values thus confirms that our method of implicit attentional manipulation was successful in directing participants' attention on specific sensory modalities.

It is important to note that the attend-visual PSS was considerably higher than the attend-auditory PSS, initially suggesting that the attend-visual condition elicited a greater attentional effect. However, it should be noted that an auditory stimulus needs to be delayed by about $40-50 \mathrm{~ms}$ compared with a visual stimulus, if the two stimuli are perceived as simultaneous. This is because of the fact that acoustic transduction time between the outer and inner ears is considerably faster than photo transduction time in the retina. ${ }^{22}$ Hence, the two psychophysical functions crossed around $-30 \mathrm{~ms}$ (audition leading vision) in Fig. 1. We did not correct it because perceptions of simultaneity are also affected by relative stimulus intensity ${ }^{13}$ and because clear criteria for matching stimuli of different modalities do not exist. ${ }^{13}$ Consequently, PSS values should be considered relative to each other and not relative to zero.

In our study, 46 participants were excluded from the data analysis, corresponding to $23 \%$ of the sample. Although this rate of exclusion appears high, it is comparable to the rates seen in similar studies: Stone et al ${ }^{14}$ excluded $26.09 \%$ of their sample because of poor task performance, and Zampini et $a l^{23}$ excluded $22.22 \%$ of their participants for the same reason. Participants who were excluded from the analysis displayed higher levels of schizotypal personality traits than those who were included. One possibility is that the excluded group had particular difficulty with sustaining attention on the task, as has generally been observed in those with schizotypal personality traits. ${ }^{24}$ High levels of schizotypy are also associated with poorer temporal sensitivity; ${ }^{15}$ which presents another reason why certain participants may have difficulties with the task.

\section{Limitations of the present study}

A potential limitation of this study is that it contained a large number of variables which led to multiple comparisons being performed in examining correlations with SPQ subscales. Because of the novel nature of this study, no statistical correction procedures to adjust for multiple comparisons were used as we wish for our results to inform future hypotheses.

A further limitation of the study was that the auditory and visual stimuli used in the TOJ task clearly differ from naturally occurring stimuli, such as speech, which affects the generalisability of these results. Emotional prosody is known to attract attention, and recent work has shown that patients with schizophrenia who experience auditory hallucinations have a greater attentional bias towards emotional sounds compared with non-hallucinating patients and healthy controls. ${ }^{25}$ Development of a similar task using speech could be a more ecologically valid approach to assess the influence of attention on perception.

\section{Future work and clinical implications}

In conclusion, we observed that highly hallucination-prone individuals and those with social withdrawal or negative features of schizotypal personality traits displayed lower levels of attention-driven auditory sensitivity compared with the rest of the sample. We proposed that this was because of an attentional bias towards internal (brain-derived) acoustic signals at the expense of external environmental signals. It is clear, however, that additional research is required to confirm and expand on our findings. Future research related to our proposal should focus on two key areas: confirming that hallucination-prone individuals have a reduced sensitivity to external stimuli under conditions of focused attention and the development of methods to investigate whether hallucination-prone individuals display enhanced internally directed attention.

If supporting evidence is found, our findings could be of relevance in the treatment of auditory hallucinations in schizophrenia. In particular, psychological therapies involving the redistribution of attentional resources may be of therapeutic benefit to some patients as an adjuvant treatment to pharmacotherapy. An appropriate treatment may be attention therapy, a technique which was originally developed as a treatment for panic disorder and anxiety and aims to direct patients' attention away from internal signals. ${ }^{26}$ There is limited evidence available concerning its efficacy in treating hallucinations in schizophrenia, although a case study of the treatment in a patient with chronic refractory auditory hallucinations provided encouraging results. ${ }^{27}$

Louise H. Rayner, MBChB, BMedSci, Kwang-Hyuk Lee, PhD, Peter W. R. Woodruff, MRCPsych, PhD, Department of Neuroscience, University of Sheffield Sheffield, UK

Correspondence: Kwang-Hyuk Lee, Department of Psychology, University of Sheffield, Sheffield S10 2TP, UK. Email: k.h.lee@sheffield.ac.uk

First received 11 Apr 2014, final revision 28 Nov 2014, accepted 15 Jan 2015

\section{Funding}

L.R. was supported by a bursary from the University of Sheffield Neurology Alumni Foundation.

\section{References}

1 De Leede-Smith S, Barkus E. A comprehensive review of auditory verbal hallucinations: lifetime prevalence, correlates and mechanisms in healthy and clinical individuals. Front Hum NeuroSci 2013; 7: 1-25.

2 Hunter MD, Eickhoff SB, Miller TWR, Farrow TFD, Wilkinson ID, Woodruff PWR. Neural activity in speech-sensitive auditory cortex during silence. Proc Natl Acad Sci USA 2006; 103: 189-94.

3 Lewis-Hanna LL, Hunter MD, Farrow TFD, Wilkinson ID, Woodruff PWR. Enhanced cortical effects of auditory stimulation and auditory attention in healthy individuals prone to auditory hallucinations during partial wakefulness. Neuroimage 2011; 57: 1154-61.

4 Neurobehavioural Systems. Presentation Version 14.9. Albany, 2011 (http://www.neurobs.com).

5 Keetels M, Vroomen J. No effect of auditory-visual spatial disparity on temporal recalibration. Exp Brain Res 2007; 182: 559-65.

6 Launay $G$, Slade $P$. The measurement of hallucinatory predisposition in male and female prisoners. Pers Individ Dif 1981; 2: 221-34.

7 Bentall RP, Slade PD. Reliability of a scale measuring disposition towards hallucination. Pers Individ Dif 1985; 6: 527-9. 
8 Raine A. The SPQ - a scale for the assessment of schizotypal personality based on DSM-III-R criteria. Schizophr Bull 1991; 17: 555-64.

9 Eysenck SBG, Eysenck HJ, Barrett P. A revised version of the psychoticism scale. Pers Individ Dif 1985; 6: 21-9.

10 Newman CW, Weinstein BE, Jacobson GP, Hug GA. The hearing handicap inventory for adults - psychometric adequacy and audiometric correlates. Ear Hear 1990; 11: 430-3.

11 Hymans DG. Curve Expert 1.4. Hixton, 2009 (http://www.curveexpert.net/ curveexpert-basic/).

12 Lee KH, Egleston PN, Brown WH, Gregory AN, Barker AT, Woodruff PWR. The role of the cerebellum in subsecond time perception: Evidence from repetitive transcranial magnetic stimulation. J Cognitive Neurosci 2007; 19 147-57.

13 Spence C, Shore DI, Klein RM. Multisensory prior entry. J Exp Psychol Gen 2001; 130: 799-832.

14 Stone JV, Hunkin NM, Porrill J, Wood R, Keeler V, Beanland M, et al. When is now? Perception of simultaneity. Proc $R$ SOC Lond B Biol 2001; 268: 31-8.

15 Lee KH, Dixon JK, Spence SA, Woodruff PWR. Time perception dysfunction in psychometric schizotypy. Pers Individ Dif 2006; 40: 1363-73.

16 Busey T, Craig J, Clark C, Humes L. Age-related changes in visual temporal order judgement performance. Vision Res 2010; 50: 1628-40.

17 Woodruff $\mathrm{P}$, Wright I, Bullmore $\mathrm{E}$, Brammer $\mathrm{M}$, Howard R, Williams $\mathrm{S}$, et al. Auditory hallucinations and the temporal cortical response to speech in schizophrenia: a functional magnetic resonance imaging study. Am $J$ Psychiatry 1997; 157: 1676-82.
18 Ford JM, Roach BJ, Jorgensen KW, Turner JA, Brown GG, Notestine R, et al. Tuning in to the voices: a multisite fMRI study of auditory hallucinations. Schizophr Bull 2009; 35: 58-66.

19 Hunter M, Woodruff P. Characteristics of functional hallucinations. Lett Am J Psychiatry 2004; 161: 923.

20 Hoffman RE, Varanko M, Gilmore J, Mishara AL. Experiential features used by patients with schizophrenia to differentiate 'voices' from ordinary verbal thought. Psychol Med 2008; 38: 1167-76.

21 Nayani T, David A. The auditory hallucination: a phenomenological study. Psychol Med 1996; 26: 177-89.

22 Alais D, Carlile S. Synchronizing to real events: subjective audiovisual alignment scales with perceived auditory depth and speed of sound. Proc Natl Acad Sci USA 2005; 102: 2244-7.

23 Zampini M, Shore DI, Spence C. Audiovisual prior entry. Neurosci Lett 2005; 381: 217-22.

24 Gooding DC, Matts CW, Rollman EA. Sustained attention deficits in relation to psychometrically identified schizotypy: evaluating a potential endophenotypic marker. Schizophrenia Res 2006; 82: 27-37.

25 Alba-Ferrara L, de Erausquin G, Hirnstein M, Weis S, Hausmann M. Emotional prosody modulates attention in schizophrenia patients with hallucinations. Front Hum Neurosci 2013; 7: 59.

26 Hatashita-Wong M, Siverstein SM. Coping with voices: selective attention training for persistant auditory hallucinations in treatment refractory schizophrenia. Psychiatry 2003; 66: 255-61.

27 Valmaggia LR, Bouman TK, Schuurman L. Attention training with auditory hallucinations: a case study. Cognit Behav Pract 2007; 14: 127-33. psychiatry in pictures

\section{High Royds Hospital, Menston, Ilkley} Richard Mindham

High Royds Hospital was the third of four large hospitals built by the West Riding of Yorkshire County Council to accommodate 'pauper lunatics'. The hospital was designed by the county engineer J. Vickers Edwards, work began in 1884 and the hospital was opened in 1888. Pavilions were arranged in an echelon formation with the administrative block, which included a tower and clock, at the centre and the service area behind, the whole linked by covered corridors. There were wards designated for the care of patients with epilepsy and other special groups. Subsequently there were many additions to the hospital which included an infirmary, long-stay wards detached from the hospital and a 'neurosis unit' built in the modern style in 1938.

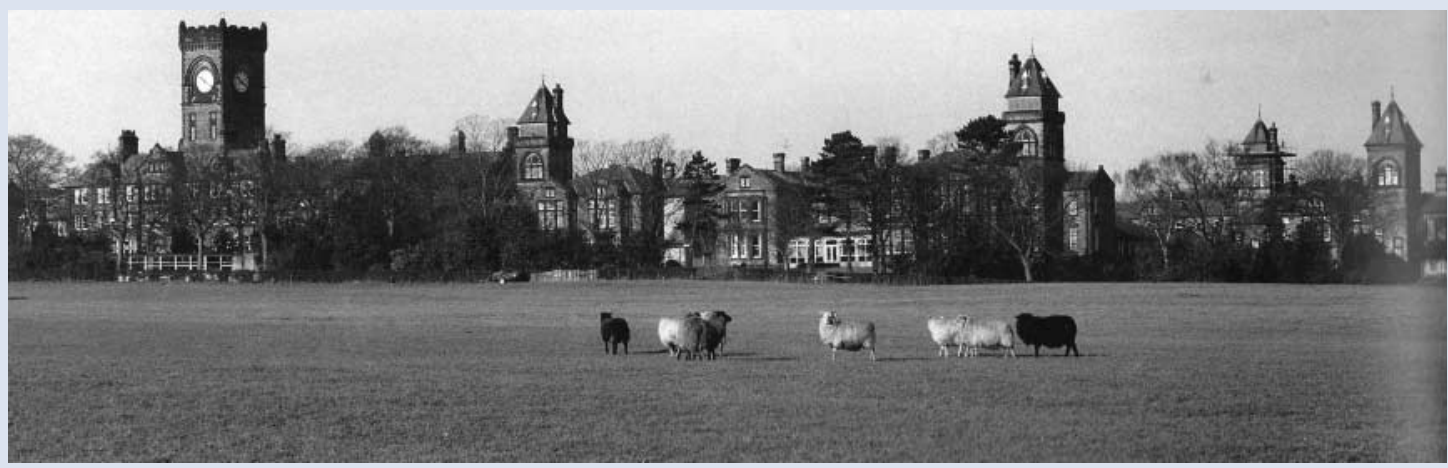

High Royds Hospital photographed by Norman Hodgson, 1995.

The hospital was set in an elevated site about 10 miles from both Leeds and Bradford, surrounded by its own extensive gardens and farm, with high moors on all sides. The hospital was built in a subdued Arts and Crafts style in sandstone from local quarries, with a splendid roof of Westmorland slate with elaborate lead work and finials. Internally, the woodwork was of oak and pitchpine, the walls were glazed to dado height and the floors were of marble mosaic. The hospital was served by its own water supply, railway line and burial ground. Facing south overlooking its grounds the hospital gave an impression of considerable grandeur.

In 1958 the hospital achieved a population of 2500 patients; thereafter it declined until its closure in 2003. It is a listed building grade $\|$ and is in a green belt. The site is being redeveloped for residential use. 